\title{
Effects of gamma $(\gamma)$-irradiation on physicochemical properties and bioavailability of iron (hydr)oxides coprecipitated with varying concentrations of organic matter
}

\author{
TAREK NAJEM AND DANIELLE FORTIN
}

\author{
University of Ottawa
}

Presenting Author: tnaje098@uottawa.ca

Organo-mineral coprecipitates formed by the association of iron (hydr)oxides and organic matter are ubiquitous in natural environments and play an important role as scavengers of contaminants as well as in the preservation of organic carbon. Such composites have been a subject of intense research for the past years to understand the impact of organic matter on the reactivity of iron (hydr)oxides, in particular their bioreducibility and adsorption kinetics/capacity of contaminants. However, one caveat in studying naturally occurring samples is that the sample must be sterilised in order to distinguish between biotic and abiotic processes. To achieve this, the sterilisation method should not only efficiently and completely eliminate microbial activity, but it should also minimally impact the physicochemical properties of the sample. One of the most common sterilisation methods described in the literature is $\operatorname{gamma}(\gamma)$-radiation. Although the impact of $\gamma$-radiation on soils and sediments in comparison to other methods has been extensively studied, very few studies have focused on its impact on the physicochemical properties of iron (hydr)oxides in relation to their organic matter content.

Therefore, in this study we investigated the impacts of $\gamma$ radiation, at a dose of $25 \mathrm{kGy}$, on the physicochemical properties of a range of wet synthetic iron (hydr)oxides (2-line ferrihydrite, lepidocrocite, and goethite) coprecipitated with varying concentrations of the polysaccharide, Na-alginate $(\mathrm{C} / \mathrm{Fe} 0,0.5$, 1.00 , and 1.5). The degree of impact was examined by using various analytical techniques as well as by assessing their bioavailability to the iron reducing bacteria Shewanella putrefaciens CN32. The results thus far show that, for the coprecipitates, $\gamma$-radiation led to the significant reductive dissolution of $\mathrm{Fe}$ as well as to the release of organic matter into solution. With respect to the bioavailability of the minerals, the rates of bioreduction did not significantly differ between the treated and untreated minerals. However, $\gamma$-irradiation significantly enhanced the extent of reduction of ferrihydritealginate coprecipitates ( $\mathrm{C} / \mathrm{Fe} \quad 0.5$ and 1.00). Despite such changes, the mineralogy across all minerals assessed did not change as determined by XRD. Our results indicate that $\gamma$ radiation induced physicochemical changes which is contrary to numerous studies identifying $\gamma$-sterilization as a minimally invasive technique. 